\title{
Estado de las investigaciones sobre la optimización en el proceso de producción de software
}

\section{Status of research on optimization in the software production process}

\section{Status da pesquisa sobre otimização no processo de produção de software}

\author{
Xavier D. Guzmán-Gutiérrez ${ }^{\mathrm{I}}$ \\ xdiazg@facinf.uho.edu.cu \\ Yosvani O. Lao-León II \\ ylaol@facinf.uho.edu.cu
}

Recibido: 26 de octubre de 2017 * Corregido: 08 de diciembre de 2017 * Aceptado: 30 de enero de 2018

I Ingeniero Informático, Profesor instructor, Facultad de Informática y Matemática, Universidad Oscar Lucero Moya, Holguín, Cuba.

II Ingeniero Industrial, Máster en Ingeniería Industrial en la mención de Producción y Logística, Profesor asistente, Facultad de Ingeniería Industrial, Universidad Oscar Lucero Moya. Holguín. Cuba. 
Dom. Cien., ISSN: 2477-8818

Vol. 4, núm. 2, abril, 2018, pp. 138-146

Estado de las investigaciones sobre la optimización en el proceso de producción de software

\title{
Resumen
}

Para optimizar cualquier proceso ya sea el de producción de software o cualquier otro debe verse de manera integral, o sea tanto el proceso en sí como su integración por otros subprocesos, esto permite realizar su optimización con la unión multidisciplinaria de las disciplinas Informática e Ingeniera Industrial. Este análisis no es muy común y requiere una búsqueda detallada de los trabajos realizados hasta ahora sobre el tema. En el presente estudio se llevó a cabo un análisis bibliométrico para sustentar la creación de una herramienta que permitirá la optimización del proceso de producción de software; el método partió de una búsqueda por palabras clave en la base de datos indexada Scopus para el posterior análisis de la bibliografía obtenida, teniendo en cuenta el año en que fue realizada, que el contenido siga la línea de investigación y los autores más destacados de esta.

Palabras clave: análisis bibliométrico; producción de software; optimización.

\begin{abstract}
To optimize any process, whether the production of software or any other must be viewed in a comprehensive manner, that this both the process itself and its integration by other sub processes, this allows its optimization with the multidisciplinary union of the disciplines Computer Science and Industrial Engineering. This analysis is not very common and requires a detailed search of the work carried out so far on the subject. In the present study, a bibliometric analysis was carried out to support the creation of a tool that will allow the optimization of the software production process; the method was based on a search by keywords in the Scopus indexed database for the subsequent analysis of the bibliography obtained, taking into account the year in which it was made, that the content follow the research line and the most outstanding authors of this.
\end{abstract}

Keywords: bibliometric analysis; software production; optimization.

\section{Resumo}

Para otimizar qualquer processo, seja a produção de software ou qualquer outro deve ser vista de forma abrangente, ou seja, o próprio processo e sua integração por outros subprocessos, isso permite sua otimização com a união multidisciplinar das disciplinas de Ciência da Computação e Engenharia 
Dom. Cien., ISSN: 2477-8818

Vol. 4, núm. 2, abril, 2018, pp. 138-146

Estado de las investigaciones sobre la optimización en el proceso de producción de software

Industrial. Essa análise não é muito comum e requer uma busca detalhada do trabalho realizado até o momento sobre o assunto. No presente estudo, foi realizada uma análise bibliométrica para apoiar a criação de uma ferramenta que permitirá a otimização do processo de produção de software; O método foi baseado em uma busca por palavras-chave no banco de dados indexado do Scopus para posterior análise da bibliografia obtida, levando em conta o ano em que foi feita, que o conteúdo segue a linha de pesquisa e os autores mais destacados.

Palavras chave: análise bibliométrica; produção de software; optimização.

\section{Introducción}

El presente trabajo pretende realizar un análisis bibliométrico con el cual sustentar, mediante métodos cuantitativos, la necesidad de optimización del proceso de producción de software. En la actualidad se acepta que la calidad del proceso de producción de software está directamente relacionada con la calidad del proceso utilizado para adquirirlo, desarrollar y mantenerlo, expresado en mejoras en el desempeño de los proyectos, aumento de la calidad de los productos obtenidos y el éxito de las organizaciones (Yanet Vega Niniet 2014), por lo que es imprescindible mantener actualizada la información en este ámbito para que el producto desarrollado pueda ser satisfactorio. Este resulta un campo de convergencia entre la Ciencia informática y la Ingeniería industrial, que aporta el enfoque científico de la calidad en la producción.

En este caso, se requirió una búsqueda de trabajos sobre el tema en una base de datos de índices bibliográficos.

Los principales índices bibliográficos, multidisciplinarios, con producción de indicadores bibliométricos son Web of Science (WoS), elaborado por la empresa Thomson Reuters, y Scopus, de la empresa Elsevier; estos índices actualizan constantemente las publicaciones ya incluidas, adicionan nuevos títulos e incorporan colecciones retrospectivas. Asociados a WoS y Scopus existen otros índices: Journal Citation Report (JCR) y SCImago Journal Rank (SJR) los cuales presentan información bibliométrica y "rankings" de las revistas indexadas. SciELO (Scientific Electronic Library Online) es un índice bibliográfico multidisciplinar y de publicación en-línea, que tiene como 
Dom. Cien., ISSN: 2477-8818

Vol. 4, núm. 2, abril, 2018, pp. 138-146

Estado de las investigaciones sobre la optimización en el proceso de producción de software

características fundamentales publicar el texto completo, en acceso abierto, y la capacidad para generar indicadores basados en la citación ya incluidos en WoS y/o Scopus, al mismo tiempo que representan la única fuente de datos de citación de aquellas revistas que aún no se encuentran indizadas en alguna de las bases de datos con producción de indicadores bibliométrico (Antonio Sánchez Pereyra 2014). Para el desarrollo de esta investigación se escogió como principal índice bibliográfico Scopus con su portal ScienceDirect.

El presente estudio representa la primera etapa de un proyecto de investigación que se lleva a cabo en la Universidad de Holguín (UHO) en la Facultad de Informática y Matemática (FACINF) y en específico en la Casa de Software (CSof), estructura encargada de brindar servicios de producción de software a empresas $\mathrm{u}$ organizaciones tanto nacionales como internacionales. Para desarrollar sus softwares, la CSof se nutre de los estudiantes de pre-grado de Ingeniería informática que cursan la carrera en dicha facultad. Los proyectos de desarrollo se gestionan solo por pedido de los organismos a la FACINF, sin embargo, esta no cuenta con un procedimiento establecido para la gestión de este proceso, ocasionando demoras y en ocasiones errores.

Los problemas encontrados sobre la gestión del proceso de producción de software indican la necesidad de investigar la posible creación de una herramienta para la optimización de este proceso, pero para ello debe profundizarse en los antecedentes y resultados de investigaciones anteriores y tenerse un análisis de los trabajos realizados sobre el tema, ello puede alcanzarse a partir del análisis bibliométrico de la producción científica en este campo, cuestión devenida en el objetivo del presente trabajo.

\section{Método}

Para obtener datos base se escogió por sus características y actualidad la aplicación online Scopus, donde se realizó una búsqueda por palabras clave realizándose tres iteraciones siguiendo la metodología TAK (búsqueda por Título, Abstract y Palabras clave): la primera buscando por proceso de desarrollo de software arrojó un total de 154 trabajos referenciados; en la segunda buscando por proceso de desarrollo de software y optimización arrojó un total de 36 referencias más y la tercera 
buscando por proceso de producción de software, optimización y reutilización arrojó 2 referencias más para un total de 192 trabajos referenciados en esta base de datos.

Los datos obtenidos fueron procesados en el software bibliográfico Endnote: El programa agrupa los datos bibliográficos en «librerías» con extensión de archivo *.enl y una carpeta correspondiente de *.data. Hay varias maneras de agregar una referencia a una biblioteca: manualmente, exportando, importando, etc. (Castilla 2009) Las referencias obtenidas de este software fueron llevadas al Excel de Windows para la posterior construcción de la red social usando el software para Windows Ucinet.

\section{Resultados}

El método propuesto comenzó con la extracción de datos de Scopus de la que se extrajeron un total de 88 trabajos los cuales seguían la línea de investigación. De ellos 79 eran artículos y 9 libros.

Dado que la mayor parte de la información encontrada son artículos de revistas, en la siguiente figura se muestran aquellas que han publicado el mayor número de trabajos relacionados. Se destaca la Procedia CIRP, en la que han aparecieron 11 artículos.

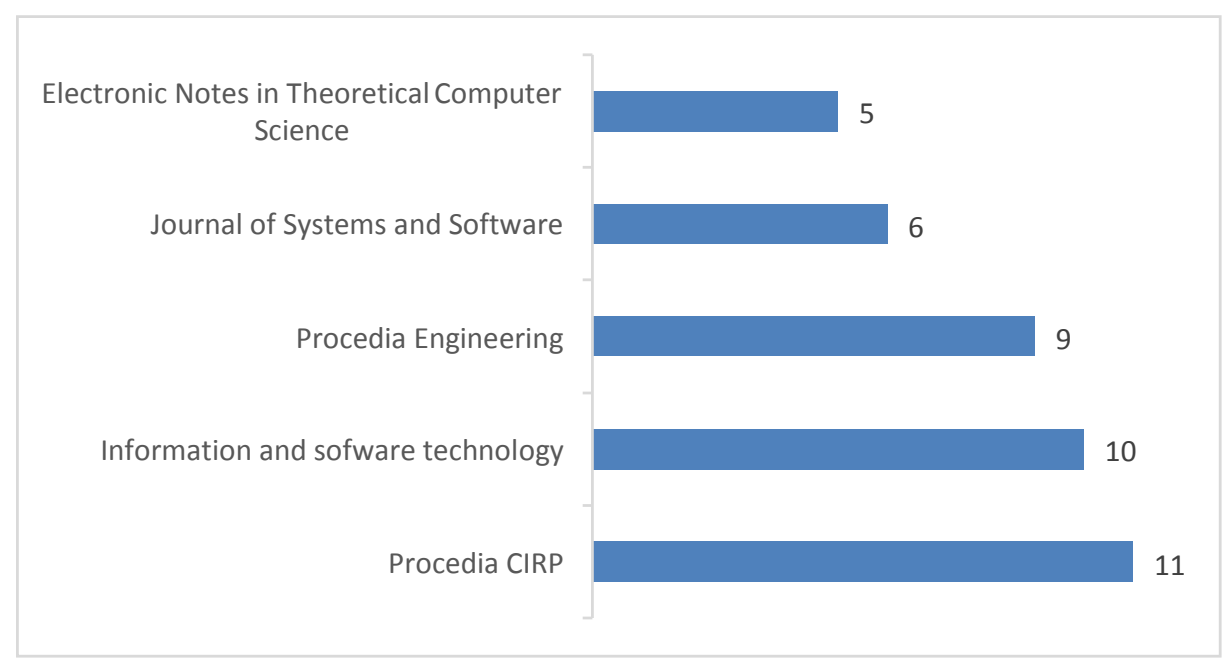

Figura 1. Revistas más importantes. 
Dom. Cien., ISSN: 2477-8818

Vol. 4, núm. 2, abril, 2018, pp. 138-146

Estado de las investigaciones sobre la optimización en el proceso de producción de software

En cuanto a la actualidad de los trabajos, se presenta un gráfico donde se aprecia que la mayor parte fueron realizados entre 2012 y 2016, aunque el tema comenzó a parecer en publicaciones periódicas desde el año 2000. En el mismo se demuestra cómo esta temática ha ido tomando relevancia, expresada en el aumento del número de publicaciones relacionadas, alcanzando la mayor frecuencia en el 2014 y en el 2016, lo que puede estar asociado al propio auge del empleo de la tecnología informática en los más diversos escenarios y con ello una creciente especialización en la producción de software, necesitada de la optimización de sus procesos de producción.

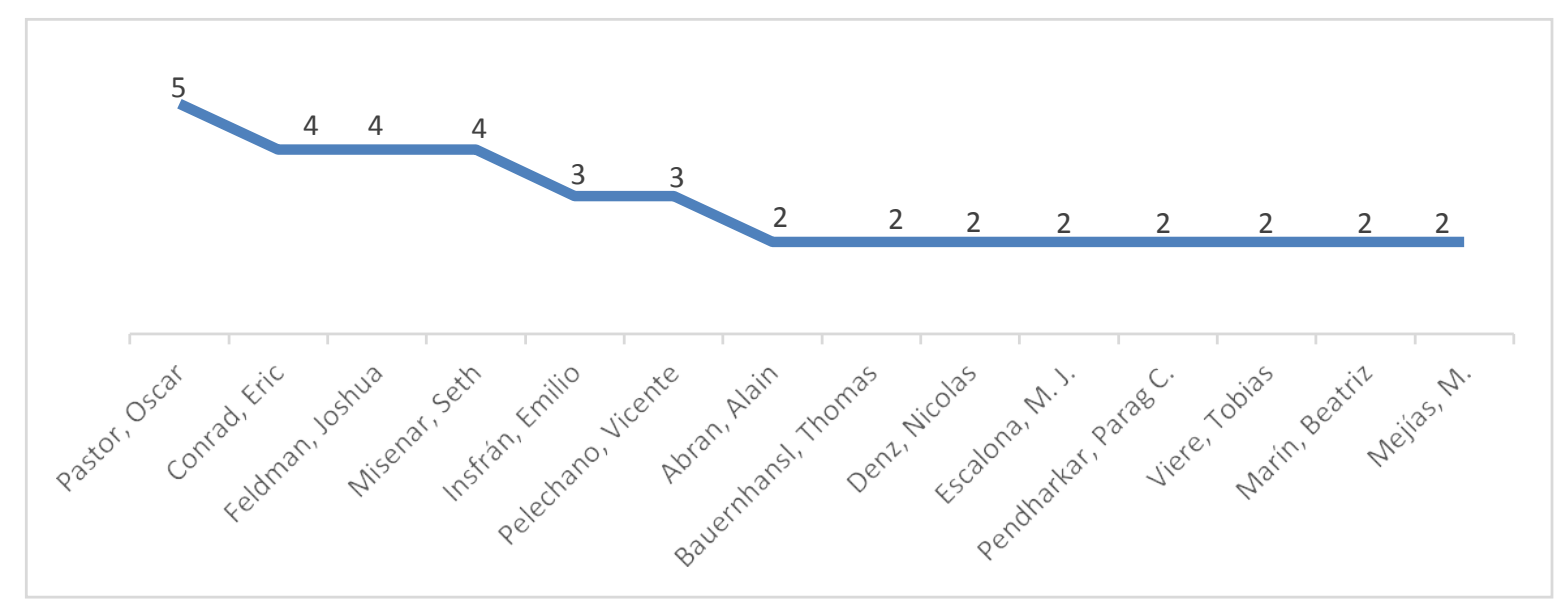

Figura 2. Cantidad de Trabajos por autor.

Las palabras clave más utilizadas se exponen mediante la siguiente figura donde se observan aquellas que fueron encontradas en al menos dos trabajos de esta bibliografía, llegando a la conclusión de que las palabras clave: optimización, simulación e ingeniería de software son las más encontradas y que estas corresponden con la línea de investigación requerida. 
Dom. Cien., ISSN: 2477-8818

Vol. 4, núm. 2, abril, 2018, pp. 138-146

Estado de las investigaciones sobre la optimización en el proceso de producción de software

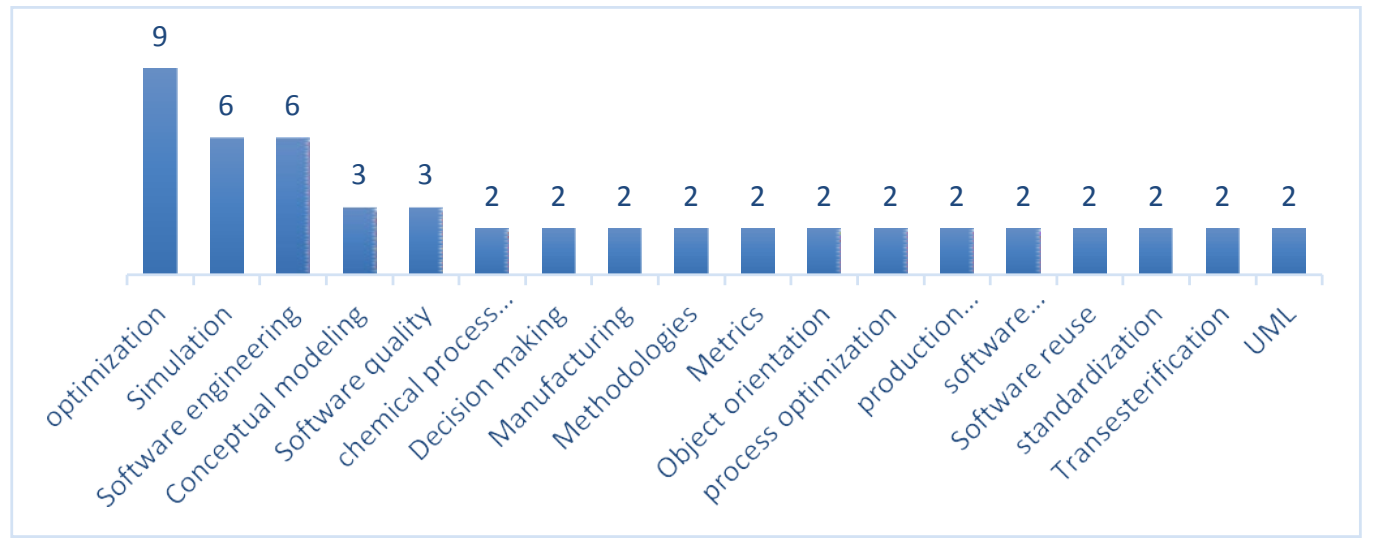

Figura 3. Palabras claves.

Para determinar la relación entre los autores que más contribuyen con la temática, se utilizó el Software para Análisis de Redes Sociales Ucinet for Windows, obteniéndose la red que se muestra en la figura. Este gráfico permite apreciar las colaboraciones de los autores por trabajos a la producción científica de otros autores resultando los más prolíficos expuestos en las siguientes figuras.

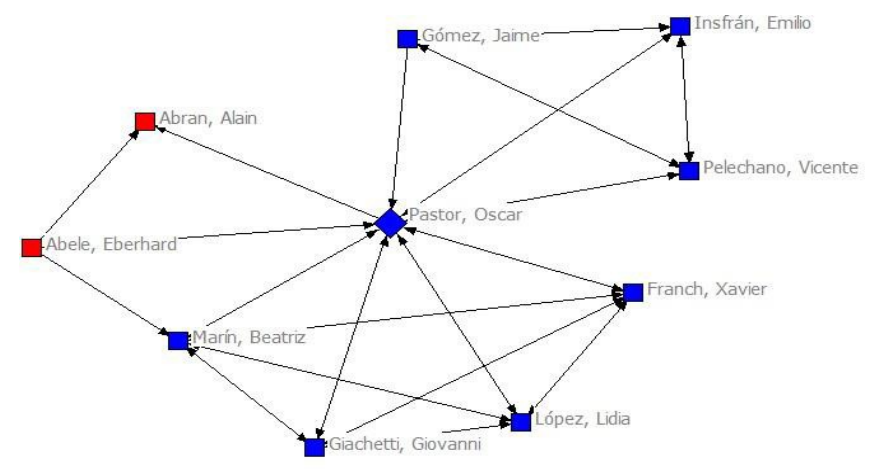

Figura 4. Redes sociales. 
Los gráficos extraídos del software Ucinet muestran una concordancia con el resto de la investigación. Autores como Oscar Pastor, Vicente Pelechano, Jaime Gómez, Emilio Insfrán, Xavier Franch, Lidia López, Giovanni Gianichetti, Beatriz Marin, Eberhard Abele y Alain Abran con sus trabajos: The OOmethod approach for information systems modeling: from object-oriented conceptual modeling to automated programming (Pastor, Gómez et al. 2001) y Verifying goal-oriented specifications used in model-driven development processes (Giachetti, Marín et al. 2017). Estos contribuyen en varios trabajos en conjunto, que siguen con la línea del tema de investigación.

\section{Análisis de indicadores}

Para conocer cuál es el autor que más se destaca de entre los que más trabajos publicaron se desarrolló un análisis de Centralidad e Intermediación conociéndose que el autor Oscar Pastor es el autor más importante para el desarrollo de esta investigación.

Tabla 1. Centralidad e Intermediación

\begin{tabular}{|l|c|c|}
\hline \multicolumn{1}{|c|}{ Autores } & Centralidad & Intermediación \\
\hline Oscar Pastor & 12.000 & 98.000 \\
\hline Abele, Eberhard & 8.000 & 52.000 \\
\hline Bauernhansl, Thomas & 8.000 & 30.000 \\
\hline
\end{tabular}

El reducido número de publicaciones científicas en el tema de la calidad del proceso de producción de software, que se demuestra en este estudio hace notar que es un campo en desarrollo, con un marcado carácter multidisciplinario, por lo que los resultados expresados aquí pueden servir de base para el desarrollo de esta línea de trabajo. 


\section{Conclusiones}

Mediante este trabajo se pudo determinar la bibliografía más actual, los autores más prolíficos y que más colaboraron entre sí en la temática de la optimización de software, por lo que constituye un instrumento de valor para la posterior creación de la herramienta que supere esas limitaciones.

La bibliografía encontrada no muestra ningún resultado de este tipo en Cuba, por lo que la investigación que se realice en esta temática constituye un aporte y un precedente para el desarrollo de este campo multidisciplinario en el país.

\section{Referencias Bibliográficas}

Antonio Sánchez Pereyra, O. C. R., Patricia Garrido Villegas (2014). Reporte bibliométrico de las revistas mexicanas de investigación. Mexico.

Castilla, R. (2009). "¿Le resulta difícil hacer la bibliografía? Los gestores de referencias bibliográficas pueden ayudarlo". Revista Acimed 19(2).

Giachetti, G., B. Marín, L. López, X. Franch and O. Pastor (2017). "Verifying goal-oriented specifications used in model-driven development processes". Information Systems 64: 41-62.

Pastor, O., J. Gómez, E. Insfrán and V. Pelechano (2001). "The OO-method approach for information systems modeling: from object-oriented conceptual modeling to automated programming". Information Systems 26(7): 507-534.

Yanet Vega Niniet, M. R. N., daima Mustelier Sanchidrian, Yademis Piñero Perez, (2014). Modelando el proceso de desarrollo de software de la UCI con cbSPEN. 